INVESTIGACIONES

\title{
Estilos de gestión pedagógica presentes en profesores de escuelas de la Región Metropolitana*
}

\author{
Styles of pedagogic management in teachers of schools in Región Metropolitana \\ Estilos de gestão pedagógica presentes em \\ professores de escolas da Região Metropolitana
}

\author{
Manuel Bastías Urra
}

Universidad Metropolitana de Ciencias de la Educación, Chile. Telf.: 02-4921256, 9-90004254.

Correo electrónico: manuel.bastias@umce.cl

\begin{abstract}
RESUMEN
Este estudio realiza una aproximación descriptiva sobre las orientaciones que enfatizan los docentes y busca identificar los estilos de gestión pedagógica que se desprenden de ellas. Para realizar la investigación se consideró los avances de la neurociencia respecto a los mecanismos cerebrales que están en la base toda acción humana, tales como la comprensión, la imaginación, el aprendizaje y otras. Aunque la muestra es pequeña, los resultados muestran que los profesores se orientan por diversas formas de pensamiento, dominando las asociadas al hemisferio cerebral izquierdo, especialmente la orientada al logro de resultados o pragmática. En algunas de las formas de aproximarse a la actividad docente se encontró que están influyendo factores personales como la edad, género y tipo de universidad de egreso. Asimismo, el análisis de la información permite inferir, por una parte, la existencia de una dinámica de cambios en la hegemonía de los paradigmas y modelos de pensamiento utilizados en el pasado $\mathrm{y}$, por otra, la emergencia de nuevas orientaciones hacia la tarea docente, más acordes con las nuevas demandas de la sociedad del conocimiento y la necesidad de formar personas versátiles e integrales.
\end{abstract}

Palabras clave: sociedad del conocimiento, educación, neurociencia, hemisferios cerebrales, gestión pedagógica.

\begin{abstract}
Following a descriptive approach this study presents the main points of view and orientations in teachers, and tries to identify the different pedagogical management styles which emerge from them. This study considers the advances in neuroscience - related to brain mechanisms - which underlie all human action, such as understanding, imagination, learning and others. Even though it is a small sample, results show that teachers have several orientations of thought, where procedures associated with the left hemisphere are dominant; in particular, those oriented to the achievement of goals or pragmatics. Likewise, the data analysis also allows us to observe, on the one hand, the existence of a dynamics of changes in the hegemony of the paradigms and models of thought used in the past, and, on the other hand, the appearance of new orientations towards the educational activity, more accurate to the demands of the knowledge society and the need to be versatile and integral individuals.
\end{abstract}

Key words: knowledge society, education, neuroscience, cerebral hemispheres, pedagogical management.

\section{RESUMO}

Por meio de uma abordagem descritiva sobre as orientações que enfatizam os docentes, visa identificar os estilos de gestão pedagógica que emergem a partir de tais orientações. Para realizar a pesquisa foram considerados os avanços da neurociência relativos aos mecanismos cerebrais que sustentam toda a ação humana como a compreensão, a imaginação, a aprendizagem, entre outros. Embora a amostra seja pequena, resultados mostram que os professores são orientados por diferentes formas de pensamento, dominando aquelas associadas ao hemisfério cerebral esquerdo, em

* $\quad$ Este estudio forma parte del proyecto DIUMCE,Fibe 01-11. 
particular, aquelas orientadas para o alcance de resultados ou pragmática. Em algumas formas de aproximar-se da atividade docente, encontrou-se que fatores pessoais como idade, gênero e tipo de graduação estão influenciando tais orientações. Da mesma forma, a análise das informações permite inferir que, por um lado, existe uma dinâmica de transformações na hegemonia dos paradigmas e modelos de pensamento antes utilizados e, por outro, surgiram novas orientações para o trabalho docente, mas consoantes com as novas demandas da sociedade do conhecimento e da necessidade de formar pessoas versáteis e íntegras.

Palavras chave: sociedade do conhecimento, educação, neurociência, hemisférios cerebrais, gestão pedagógica.

\section{INTRODUCCIÓN}

La nueva realidad que está emergiendo como resultado de los fenómenos de la globalización, masificación de las tecnologías de la información y, especialmente, la sociedad del conocimiento, tiene como característica ser cada vez más compleja, incierta, competitiva y dinámica, donde los procesos de transformación política, social, cultural, económica e institucional son cada vez más veloces y altamente dependientes de nuevos factores intangibles como el conocimiento, la sensibilidad, los valores y la innovación.

Este nuevo contexto pone en entredicho a varias concepciones y fórmulas de desarrollo conocidas hasta ahora $\mathrm{y}$, consecuentemente, plantea la necesidad de introducir transformaciones sustantivas en las instituciones y sus prácticas. En el ámbito educativo, las organizaciones escolares deberán tomar distancia de los paradigmas y modelos impulsados durante la era industrial que aportaron significativamente a la configuración del tipo de organización educativa que hoy conocemos. Asimismo, los profesionales de la educación deberán transformar sus prácticas, siendo necesario para ello el abandono de la hegemonía del paradigma racionalista, perspectiva que concebía la esencia del ser humano sólo en la razón, lo cual posibilitará la incorporación de otras formas de pensamiento y actuación, más allá de las orientaciones racionales, lineales y convergentes.

En este estudio se explora las posibles dinámicas de cambio en las orientaciones de los docentes hacia su práctica profesional y, específicamente, en los estilos de gestión pedagógica presentes en una muestra de 51 docentes de 7 escuelas de la provincia de Santiago de la Región Metropolitana.

\section{CONFIGURACIÓN DE LA PRÁCTICA EDUCATIVA DURANTE LA ERA INDUSTRIAL}

A lo largo de la historia de la humanidad la educación fue un acto muy simple y siempre presente, pues bastaba con delegar la tarea de educar en aquellas personas con mayores habilidades o conocimientos. Sólo en el siglo XVII el Estado Prusiano creó las primeras escuelas y el acto de educar se confinó al aula, mediante una enseñanza muy rudimentaria que tenía como finalidad enseñar a leer, escribir y a utilizar algunas operaciones matemáticas básicas a una vasta población campesina analfabeta (Palacios, 1991). El cambio más notable en la tendencia mundial a la escolarización se produjo a partir de la segunda mitad del siglo XVIII con el inicio de revolución industrial, acontecimiento que empezó a demandar a las rudimentarias instituciones educativas existentes algunas transformaciones que les permitieran responder a las nuevas necesidades provenientes del trabajo productivo fabril (Alighiero, 1976), especialmente formando 
recursos humanos preparados para insertarse en ambientes de producción mecanizada, cumpliendo largas jornadas de trabajo en puestos específicos y con funciones laborales muy acotadas

Como resultado de la creciente alianza entre la escuela y las necesidades de los procesos productivos fabriles, se avanzó gradualmente hacia la consolidación de un tipo de institución educativa que Toffler ha llamado "escuelas fabriles" (Toffler, 1980: 335), denominación que busca expresar la creciente simetría, en forma y contenido, entre las instituciones escolares y las fábricas, lo cual se puso de manifiesto en distintas dimensiones de la escuela como: el tipo de arquitectura; la forma de gestionar la organización; el currículo de enseñanza; el tipo de pensamiento estimulado; la cultura escolar; las prácticas de enseñanza y otros aspectos presentes en el mundo escolar de las sociedades industriales (Aparici, 1996).

A partir de esta relación, uno de los énfasis de las instituciones escolares fue encontrar aquellas fórmulas pedagógicas que permitieran transferir el conocimiento considerado como verdadero, de acuerdo con la propuesta positivista impulsada por Comte (Comte, 1982), es decir, identificar aquellos mecanismos que facilitaran el traspaso a las mentes de los alumnos de conocimientos racionales, objetivos, formales y sistemáticos. Esta propuesta consideró que la educación era fundamentalmente un acto puramente intelectual $\mathrm{y}$, en consecuencia, se debía procurar el desarrollo de una forma de pensamiento que permitiera a los alumnos contar con las capacidades para analizar y resolver situaciones de manera racional, es decir, mediante procedimientos lógicos y analíticos. Desde esta perspectiva, en la tarea educativa se enfatizó el uso de procedimientos codificados tales como textos, palabras, dibujos, datos y otros medios de transmisión de información o conocimiento explícito (Nonaka, 1999).

A comienzos del siglo XX y una vez consolidada la producción industrial, aparece el pensamiento administrativo clásico cuyo principal exponente es Taylor (Taylor, 1981), quien propugnó el desarrollo de organizaciones racionales, es decir, que el trabajo al interior de ellas se desarrollara de acuerdo a principios científicos y de esta manera alcanzar la máxima eficiencia. La metáfora de la organizaciones como máquinas (Morgan, 1996) también fue adoptada por las organizaciones escolares las que, gradualmente, se empezaron a concebir como máquinas de procesar y transmitir información. Siguiendo esta tendencia mecanicista se empieza a promover que el trabajo docente se desarrollara en forma aislada, en espacios cerrados y privados. Asimismo, se buscó homogenizar las actividades docentes, mediante mecanismos estandarizados como el uso de programas, manuales y exámenes regulados.

El sustento paradigmático de la civilización industrial fue alterado a partir de la segunda mitad del siglo XX, mediante la incorporación del concepto racionalidad instrumental. Fue Max Weber quien, en Economía y Sociedad (Weber, 1996), conceptualizó la existencia de distintos tipos ideales de acción y los asoció a distintos tipos de racionalidades, argumentando que el tipo de acción racional correspondiente a las sociedades avanzadas y modernas era aquella forma de pensamiento que está absolutamente desprovista de emociones, sentimientos y otras distorsiones humanas. Para Weber la acción orientada a lograr una finalidad calculada (racionalidad con arreglo a fines), o sea, aquella forma de pensamiento que permite lograr el mejor resultado, con el menor costo y tiempo, representa la racionalidad suprema. Desde esta postura se comenzó a impulsar la existencia de organizaciones eficientes y exitosas, donde sus 
miembros se sometieran a la lógica de la relación tecnológica con el medio a través de un control metódico, calculador y calculado (Rodríguez, 2003). La fuerza que adquirió la noción de racionalidad instrumental, acompañada por el optimismo en los procesos modernizadores, la fe en el progreso de la humanidad y la confianza en la tecnología, llevó a que esta orientación llegara a ser la predominante no sólo en las actividades de la esfera que le es propia, la económico-productiva, sino también alcanzara gran influencia en otras esferas de actividad humana, a medida que las políticas neoliberales fueron hegemonizándose, como la simbólico-cultural que es ámbito donde se despliega la educación.

En el mundo educativo el modelo de la eficacia adquiere gran relevancia a partir de la década de los 80, con la emergencia de la noción de calidad en educación. Esta nueva orientación implicó un desplazamiento desde el tiempo de permanencia en la escuela hacia los aprendizajes adquiridos por los estudiantes, situación que supone la posibilidad de un posterior desempeño laboral de excelencia y aumentar sus ingresos (Hanushek, 2007). Un aspecto central en esta propuesta es que pone como requerimiento la medición de los rendimientos educativos mediante sistemas evaluativos nacionales que, a través de pruebas estandarizadas, permitan medir aquellos logros cognitivos que son considerados básicos para el desempeño en ambientes laborales tales como el cálculo, la lectura, la escritura, las ciencias físicas y naturales.

Una consecuencia práctica de esta nueva orientación es que la racionalidad instrumental empieza a ser promovida, desde la política pública, como una nueva forma de pensamiento y actuación que debe ser incorporada por el profesorado para guiar los procesos de análisis, organización y evaluación de sus prácticas (Bastías, 2011). Una dificultad con esta forma de pensamiento calculador e instrumental es que promueve una visión de la profesión docente como una actividad técnica, altamente especializada, fragmentaria e impersonal (Popkewitz, 1990), y se corre el riego de devaluar la importancia que tienen otras formas de pensamiento humano que son las que posibilitan crear espacios de convivencia y comunicación con otros, reflexionar sobre los sentidos, generar innovaciones, activar valores, obtener nuevas comprensiones y construir conocimiento propio.

En síntesis, a partir de los procesos de industrialización, las prácticas educativas empezaron a orientarse por fórmulas inspiradas en el modelo cognitivo cartesiano, basado en la razón desprovista de toda emotividad, lo que trajo consigo la concepción dualista hombre-mundo, es decir, la existencia de un sujeto puro que conoce y un mundo exterior estable que puede ser descrito y explicado. Luego, con el avance del siglo XX, el desarrollo organizacional de las instituciones educativas fue adscribiéndose al modelo racionalista de la administración, es decir, las instituciones educativas se comenzaron a estructurar buscando funcionar con precisión, con división de funciones especializadas, sin lugar para la corporalidad, las emociones, la imaginación y otros atributos humanos visualizados como no deseables por el modelo científico de la administración. Finalmente, el ciclo racionalista se cierra con la propuesta weberiana acerca de la supremacía que tiene la racionalidad instrumental, forma de pensamiento que subordina los fines a los medios, donde lo importante es el éxito, la eficacia y la utilidad. Esta orientación propugna que toda persona debe tener las capacidades para desarrollar acciones estratégicas, utilizando una serie de procedimientos lógicamente calculados y organizados, en función de lograr determinados fines. 


\section{LA EDUCACIÓN EN EL UMBRAL DE LA SOCIEDAD DEL CONOCIMIENTO}

Así como en su época el notable intelectual cubano José Martí planteó "En tiempos teológicos, universidad teológica. En tiempos científicos, universidad científica" (cit. por Agramonte, 1991: 56), la sociedad del conocimiento también requerirá un tipo diferente de institución educativa y nuevas prácticas pedagógicas, lo cual implica considerar otras formas de pensamiento y actuación que estén en mayor sintonía con las inciertas, dinámicas y complejas realidades del futuro (Morín, 1996). El término "sociedad del conocimiento" surgió en los años 90 como un concepto alternativo a la "sociedad de la información", el cual estaba asociado a la innovación tecnológica y contiene un sentido integral y transformador pues, como lo ha señalado la UNESCO, no se relaciona únicamente con la dimensión económico-productiva sino que tiene sentido al interior de procesos de transformación política, social, cultural, económica, e institucional (UNESCO, 1994). Esta emergente nueva realidad ha acentuado el proceso de cuestionamiento y erosión de las bases epistemológicas sobre las cuales se configuraron la escuela y prácticas educativas durante la era industrial. En lo que sigue, examinaremos algunas de los cambios ocurridos en la matriz epistemológica que sustentó la práctica escolar durante la civilización industrial.

La primera reacción al dominio del modo de pensar lógico-positivista ocurrió a finales del siglo XIX con Dilthey (1978), quien desarrolló una propuesta hermenéutica que puso el énfasis en la comprensión (Verstehen), propugnando que lo central en la vida humana no es la explicación llevada a cabo mediante procesos puramente intelectuales sino la comprensión, actividad en la cual el sujeto debe emplear todas sus facultades mentales. Una característica de la comprensión (o entendimiento) es que siempre se realiza desde el particular horizonte del sujeto que intenta aprehender un fenómeno, considerando su propia historicidad y temporalidad. Posteriormente y gracias a los aportes de la fenomenología, inaugurada con Husserl en 1910 y desarrollada posteriormente por Heidegger, Gadamer y Merlau Ponty entre otros, se inició un trazado que privilegia la interpretación, el análisis crítico y la comprensión de los fenómenos o situaciones por sobre la mera explicación (Ferraris, 2002). En el campo educativo, y distanciándose de la concepción instruccional de la enseñanza, emergieron notables pensadores como Ausubel, Bruner, Vygotsky y otros, quienes propugnaron que los procesos de aprendizaje no se logran con la mera transmisión de contenidos, sino con procesos donde el sujeto es productor de sus propios conocimientos (Carretero, 2005).

En las instituciones educativas de la era industrial, dada la orientación hacia formas de pensamiento racional, lineal y convergente, en su quehacer no tuvieron cabida otros marcos epistémicos que no se ajustaran a procesos de conocimiento racionales, objetivos, formales y sistemáticos. Esta aproximación tuvo como consecuencia la exclusión del pensamiento divergente, la estigmatización del error, la represión a la originalidad y la negación de las emociones. Sin embargo, ya en el año 1951 Guilford estableció la distinción entre el pensamiento convergente, asociado al racionalismo, y el divergente, que permite procesos mentales creativos (Guilford, 1983). Otra reacción al dominio hegemónico del pensamiento lógico provino de parte de De Bono, quien acuñó el término pensamiento lateral para diferenciarlo del pensamiento lógico. Según este autor, "Si la mente humana trabajara como una biblioteca, la información nueva se almacenaría simplemente en los estantes vacíos, sin intención alguna de incorporarla al sistema existente" (De Bono, 
1994: 47). En consecuencia, en un mundo que presenta realidades complejas, problemas y situaciones inéditas, las personas que utilicen solamente el pensamiento lógico, sin creatividad, estarán limitando sus posibilidades de acción puesto que, generalmente, encontrarán una única respuesta al problema enfrentado, generalmente la convencional.

Otra importante contribución a erosionar las bases epistemológicas de la civilización industrial fueron los aportes de la sociolingüística y su aproximación al lenguaje con la intención de superar la concepción positivista, que lo concibió como un simple medio o herramienta para describir la realidad. De acuerdo a Echeverría (2008), fue Austin uno de los primeros que señaló que lo más importante del lenguaje no es representar lo existente sino que lo central es la acción misma que posibilita el acto lingüístico, es decir, el lenguaje tiene un poder transformador pues posee la virtud de poder generar identidades, relaciones sociales, compromisos, posibilidades futuras y mundos distintos. En el ámbito educativo, el nuevo modo de concebir el lenguaje ha llevado al gradual abandono de la metáfora del tubo, centrada en la transmisión de información a audiencias pasivas y comprender que lo central son los actos comunicacionales que se pueden gatillar mediante la creación de eventos donde la palabra es sólo un componente de procesos que son mucho más integrales, pues incorporan el ser total con sus pensamientos, emociones, intenciones y corporalidad. Se puede afirmar, entonces, que en todo acto educativo donde existan procesos comunicativos integrales entre los sujetos participantes se produce un proceso de elaboración, de interpretación, de significación y construcción de realidades.

Otra perspectiva sujeta a cambio fue la tendencia tradicional a relacionar la inteligencia con la capacidad de raciocinio lógico, replanteamiento que estuvo a asociado a la emergencia del concepto de inteligencia emocional, definida como la habilidad del sujeto para percibir y considerar las emociones, tanto positivas como negativas, de forma adecuada, la capacidad para comprenderlas correctamente y la pericia para regular y modificar tanto el propio estado de ánimo de sí mismo como del otro (Goleman, 1997). Esta nueva perspectiva sugiere que a las personas, para insertase en los complejos, dinámicos y cambiantes escenarios que caracterizarán el futuro, no les serán suficientes sólo el dominio de saberes racionales, especializados e instrumentales. Para los sujetos de las sociedades post-industriales será clave contar, además, con otras capacidades llamadas "blandas genéricas" como el pensamiento crítico, la resilencia, la sociabilidad, el trabajo en redes, las habilidades de comunicación, la inteligencia emocional, la activación de valores y otras capacidades básicas (Brunner, 2000).

Otro valioso aporte para superar la supremacía de las teorías racionalistas son los estudios realizados por la neurociencia cognitiva, con su interés por comprender el cerebro, su estructura y sus funciones, lo cual ha permitido valorar la importancia que tienen otras formas de cognición y el desarrollo de diversas habilidades personales. La neurociencia afirma que existen funciones mentales vinculadas al hemisferio izquierdo del cerebro, asociadas al pensamiento lógico racional y otros asociadas al hemisferio derecho, donde predominan las emociones, el pensamiento intuitivo y la imaginación creativa (Verlee, 1995). Lo más importante que señala esta propuesta es que, aunque ambos hemisferios cerebrales son diferentes, los dos utilizan modos de cognición de alto y complicado funcionamiento mental y que los procesos cognitivos se verán limitados si ambos hemisferios no están implicados (OECD, 2009).

En síntesis, asistimos a la emergencia de una nueva etapa societal que se caracterizará por la incertidumbre, la complejidad y el cambio permanente, donde las formas de 
pensamiento y actuación que fueron propiciadas durante la era industrial serán insuficientes para desenvolverse adecuadamente en las sociedades del tercer milenio. En la superación de los modelos elaborados durante la civilización industrial se han realizado importantes aportes provenientes desde distintas disciplinas, los cuales han tenido como proyecto compartido superar el reduccionismo del paradigma lógico-analítico que orientó la configuración de las instituciones y el quehacer intelectual y práctico durante la civilización industrial. Es probable que la emergencia de la sociedad del conocimiento también esté siendo acompañada por algunas transformaciones al interior de las instituciones educativas, teniendo como soporte los aportes de nuevos enfoques, como los mencionados anteriormente y otros. Uno de los posibles cambios en las instituciones educativas dice relación con las orientaciones de los docentes hacia su actividad y estilos de gestión pedagógica.

\section{ESTILOS DE GESTIÓN PEDAGÓGICA}

Cada vez hay más consenso que los centros educativos, en el contexto de la sociedad del conocimiento y del riesgo, tendrán que ser instituciones ágiles, capaces de relacionarse proactivamente con entornos inciertos y turbulentos, con prácticas permanentes de innovación y cambio organizacional, con docentes dotados de habilidades académicas y personales que les permitan promover procesos de pensamiento de todo tipo e inducir procesos integrales de producción de conocimiento, con estudiantes que posean capacidades de iniciativa, pensamiento autónomo, trabajo colaborativo, creatividad, comunicación eficaz y convivencia apoyada en valores. De allí la importancia que adquiere en los procesos educativos el estilo de gestión que utilicen los docentes en su aproximación a la actividad pedagógica.

Así como cada persona configura una forma o estilo particular de establecer relación con el mundo (Salas, 2008; SEP, 2004), también cada docente establece su propio método o conjunto de estrategias de intervención educativa, apoyado en los paradigmas, teorías, modelos y supuestos que circulan en el ámbito educativo. Estas orientaciones o tendencias a enfatizar algunas determinadas formas de pensamiento y actuación constituyen un particular estilo de gestión pedagógica el cual está asociado a ciertas habilidades personales específicas. Cada modo de orientación es también el resultado de la combinación de factores personales como, por ejemplo, la propia experiencia escolar, la formación docente, edad, género y experiencia en el ejercicio de profesión. Asimismo, la forma de aproximarse a la actividad docente no es estática ya que puede evolucionar y cambiar, de la misma forma que lo hacen los supuestos epistemológicos, las realidades y las demandas del entorno hacia la educación.

Al realizar una revisión documental acerca de los modelos y teorías relacionadas con las funciones cerebrales, se afirma que existen determinadas habilidades que están relacionadas con el tipo de orientación mental del sujeto (Verlee, 1995). Así, una persona puede sobresalir en habilidades relacionadas con un área del cerebro o también puede tener desarrolladas habilidades relacionadas con distintas áreas. De acuerdo a lo anterior y realizando una adaptación del modelo elaborado por David Kolb (Kolb, 1984), podemos identificar cuatro tipos de gestores típicos: el analítico, el pragmático, el socio-dinámico y el creativo. 
El gestor analítico ser caracteriza por ser abstracto, sistemático, visionario, convergente, sistemático, informado y otras habilidades que le permiten examinar, distinguir y aislar las partes de un todo hasta llegar a conocer los principios o elementos particulares de una situación. Las habilidades de este tipo de gestor están asociadas al hemisferio izquierdo del cerebro y son las que le permiten desarrollar conceptos e ideas que tengan un sustento racional y lógico.

El gestor pragmático tiene como características el ser detallista, metódico, calculador, disciplinado y otras habilidades que le posibilitan seleccionar el curso de acción más adecuado, considerando la información disponible y estableciendo las estrategias para orientar el proceso a seguir, a fin de lograr los objetivos considerados con eficacia y eficiencia. Este tipo de habilidades están asociadas al hemisferio izquierdo del cerebro y son las que le permiten orientarse hacia la acción con resultados terminales.

El gestor socio-dinámico se caracteriza por ser realizador, líder, arriesgado, afectivo, expresivo y otras habilidades que le permiten interactuar con otros y con el mundo que le rodea en forma activa, adecuada y positiva. Las habilidades de este tipo de gestor están vinculadas al hemisferio derecho del cerebro y son las que le permiten implicarse emocionalmente, liderar y manejar adecuadamente las emociones en diversas situaciones.

El gestor creativo tiene como características el ser imaginativo, intuitivo, sensible, comprometido, independiente, paciente y otras habilidades que lo facultan para descubrir lo nuevo o inusual. Estas habilidades están vinculadas al hemisferio derecho del cerebro y son las que le permiten incorporarse a procesos de exploración y descubrimiento de soluciones no convencionales.

Finalmente, cabe señalar que la hipótesis de trabajo de este estudio sostiene que los estilos de gestión pedagógica utilizados por los profesores están preferencialmente vinculados a formas de pensamiento racional, lineal y convergente, es decir, emplean estilos de gestión asociados al hemisferio izquierdo del cerebro.

\section{METODOLOGÍA DEL ESTUDIO}

Para realizar este estudio exploratorio, cuyo interés teórico y práctico fue identificar los estilos de gestión pedagógica que utilizan los profesionales de una muestra de instituciones educativas, se utilizó una adaptación del inventario de Kolb y que fue validado con estudiantes de magister en gestión educacional, instrumento que permite determinar cuatro escalas de orientación a partir de dos ejes: concreto-abstracto y reflexión-acción. El cuestionario propiamente tal contiene 10 líneas de 4 palabras cada una y el encuestado debe, considerando las actividades docentes que habitualmente realiza en la institución, otorgar un valor a cada uno de los cuatro términos de cada línea, asignado el valor 1 a la palabra que menos le caracteriza y 4 al término que más lo caracteriza. Desde las respuestas se puede calcular los valores obtenidos en cada una de las cuatro escalas de orientación, es decir, conocer el grado en que el sujeto se orienta hacia la Experiencia Concreta (EC), la Observación Reflexiva (OR), la Conceptualización Abstracta (CA) y la Experimentación Activa (EA). Los valores posibles de obtener en cada escala pueden oscilar en 0 y 28 puntos:

- Escala EC: Los sujetos que obtienen altos valores en esta escala están más orientados hacia la experiencia concreta y tienden a enfatizar el juicio intuitivo. 
- Escala CA: Los sujetos que obtienen altos valores privilegian la conceptualización abstracta y enfatizan el análisis racional y la abstracción.

- Escala EA: Estos sujetos con altos valores en esta escala tienden a orientarse hacia la experimentación activa, privilegiando la realización de tareas concretas y la búsqueda de alternativas innovadoras

- Escala OR: Los sujetos que obtienen altos valores en esta escala están más orientados hacia la observación reflexiva y tienden a aproximarse a las situaciones que enfrentan de manera imparcial y reflexiva.

Luego que los participantes obtuvieron los valores para cada una de las cuatro escalas de orientación, se les solicitó calcular los valores para cada eje mediante las sustracciones de los valores obtenidos (CA - EC) y (EA- OR), lo cual les permitió situarse en alguno de los cuadrantes que contienen las categorías de estilos de gestor que utilizamos en este estudio (ver Gráfico 1). Asimismo, se recogió la opinión de los docentes acerca de si el estilo obtenido correspondía a su propia percepción, respuestas que fueron afirmativas en la totalidad de los casos.

\section{Grafico 1. Estilos de Gestores}

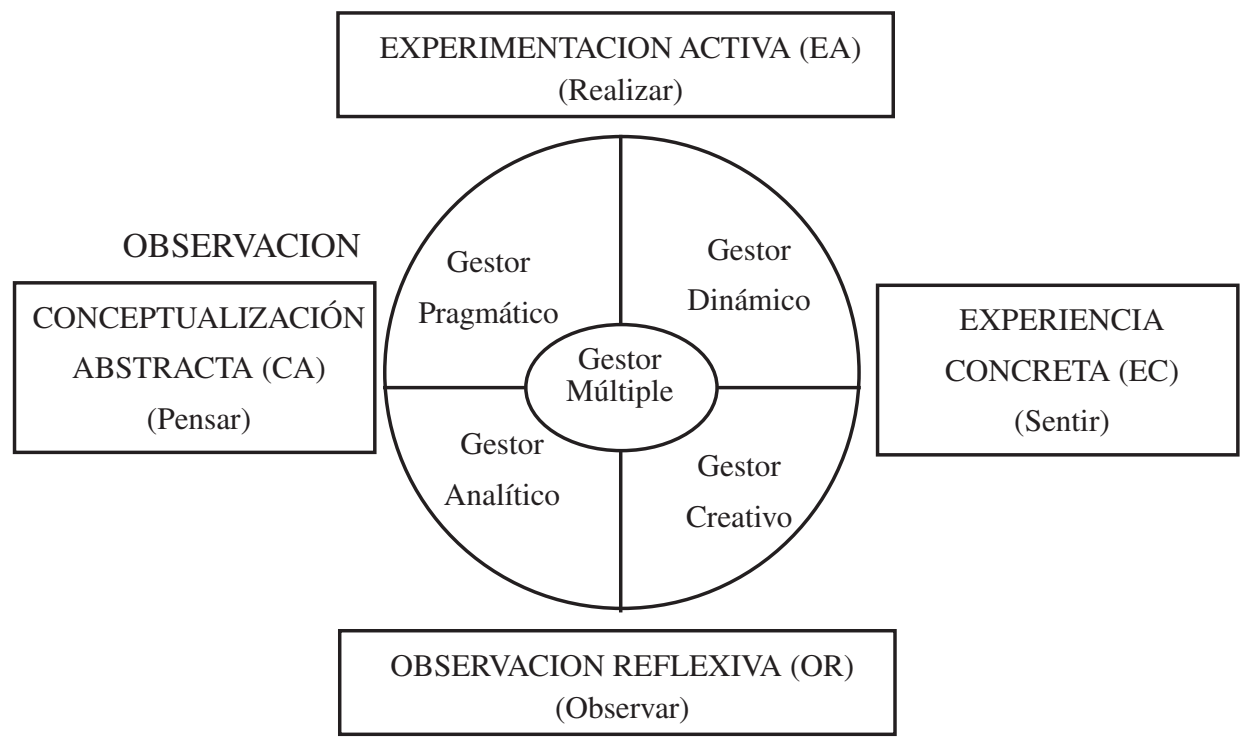

1. El Gestor Dinámico se aproxima al conocimiento y a la actividad a través de la experimentación activa y la experiencia concreta. Se caracteriza por su interés en identificar las necesidades de personas o grupos, implicarse afectivamente en las situaciones y utilizar el sentido común para llevar a cabo iniciativas de acción.

2. El Gestor Creativo se aproxima a través de la experiencia concreta y la observación reflexiva. Se caracteriza por ser original, imaginativo e intuitivo y se interesa, antes de generar una estrategia de acción específica, por conocer otras experiencias, opiniones y puntos de vista. 
3. El Gestor Pragmático se aproxima a través de la conceptualización abstracta y la experimentación activa. Se caracteriza su orientación hacia la aplicación práctica de conceptos e ideas y tratar de lograr resultados concretos, mediante procesos racionales, ordenados, estructurados y planificados.

4. El Gestor Analítico se aproxima a través de la conceptualización abstracta y la observación reflexiva. Se caracteriza por su capacidad para elaborar conceptos e ideas lógicas debidamente pensadas, que luego utilizan para enfrentar las situaciones en que se vea involucrado.

5. El Gestor Múltiple. En este estudio se consideró esta quinta categoría, debido a que un número importante de docentes distribuyó sus respuestas entre las cuatro escalas de orientación dando como resultado quedar localizados en el centro del círculo del modelo. Este gestor múltiple o polifacético, entonces, se caracteriza por aproximarse a experiencia profesional empleando, en forma combinada, formas de pensamiento y actuación vinculadas a ambos hemisferios.

Después de la autoaplicación del instrumento se solicitó a los profesores completar una ficha con sus antecedentes personales como edad, sexo, universidad de egreso, disciplina y otros. A partir de los datos obtenidos desde la aplicación de los cuestionarios y la información proporcionada en las fichas de antecedentes, se realizó un análisis estadístico descriptivo utilizando el programa SPSS18 para PC. Este análisis está basado principalmente en frecuencias y porcentajes.

\section{MUESTRA}

Para realizar el estudio se seleccionó una muestra no probabilística intencionada de 7 establecimientos educacionales de la Provincia de Santiago en la Región Metropolitana y se solicitó a los profesionales, con al menos 5 años de experiencia, que voluntariamente se ofrecieron para participar en el estudio, auto aplicarse el instrumento bajo la supervisión del encargado del estudio. En total participaron 51 profesores de escuelas de las comunas de Macul, Ñuñoa, Peñalolén, El Bosque y Batuco (Tabla 1). Los sujetos participantes en el estudio son mayoritariamente de sexo femenino $(85,7 \%)$. Todas las escuelas son municipales a excepción de una particular subvencionada.

Tabla 1. Participantes en el estudio por tipo de institución, comuna y sexo

\begin{tabular}{|c|c|c|c|c|c|}
\hline & Tipo de Institución & Comuna & Mujeres & Hombres & Total \\
\hline 1 & Municipal & Peñalolén & 12 & 4 & 16 \\
\hline 2 & Municipal & Peñalolén & 8 & 1 & 9 \\
\hline 3 & Municipal & El Bosque & 7 & 4 & 11 \\
\hline 4 & Municipal & Ñuñoa & 1 & 1 & 2 \\
\hline 5 & Municipal & Macul & 3 & 0 & 3 \\
\hline 6 & Municipal & Macul & 4 & 0 & 4 \\
\hline 7 & Particular subvencionada & Batuco & 4 & 2 & 6 \\
\hline \multicolumn{3}{|c|}{ Total } & 39 & 12 & 51 \\
\hline
\end{tabular}


Los 51 profesores de la muestra se distribuyen proporcionalmente con rangos de edades que fluctúan entre menos de 30 años y más de 61 años (Tabla 2).

Tabla 2. Edades de los participantes en el estudio

\begin{tabular}{|c|c|c|}
\hline Edades & Frecuencia & $\%$ \\
\hline Menos de 30 años & 13 & 25,5 \\
\hline $31-40$ & 11 & 21,6 \\
\hline $41-50$ & 15 & 29,4 \\
\hline $51-60$ & 10 & 19,6 \\
\hline Más de 61 años & 2 & 3,9 \\
\hline Total & 51 & 100 \\
\hline
\end{tabular}

La muestra incluye profesionales de la educación egresados de distintas universidades y representan a 13 especialidades. Más de la mitad de la muestra $(50,9 \%)$ está integrada por profesores de 3 especialidades: Matemáticas, Primer Ciclo de la Educación General Básica (NB1) y Lenguaje (Tabla 3). Casi la mitad de los participantes en el estudio $(45,1 \%)$ egresó de universidades estatales, un $43,1 \%$ cursó sus estudios en universidades privadas y un $11.8 \%$ se tituló en universidades privadas pertenecientes al Consejo de Rectores de las Universidades Chilenas (CRUCh).

Tabla 3. Especialidades de los profesores de la muestra por tipo de universidad

\begin{tabular}{|c|c|c|c|c|c|c|c|c|}
\hline & \multicolumn{2}{|c|}{$\begin{array}{c}\text { Universidad } \\
\text { Pública }\end{array}$} & \multicolumn{2}{c|}{$\begin{array}{c}\text { Privada } \\
\text { Cruch }\end{array}$} & \multicolumn{2}{c|}{ Privada } & \multicolumn{2}{c|}{ Total } \\
\cline { 2 - 9 } & Frec. & $\%$ & Frec. & $\%$ & Frec. & $\%$ & Frec. & $\%$ \\
\hline Matemáticas & 6 & 26,1 & 2 & 33,3 & 2 & 9,1 & 8 & 19,6 \\
\hline Nivel Básico 1 & 5 & 21,7 & 3 & 50,0 & 1 & 4,5 & 9 & 17,6 \\
\hline Lenguaje & 3 & 13,0 & - & - & 4 & 18,2 & 7 & 13,7 \\
\hline C. Naturales & 3 & 13,0 & - & - & 2 & 9,1 & 5 & 9,8 \\
\hline Historia & 1 & 4,3 & 1 & 16,7 & 2 & 9,1 & 4 & 7,8 \\
\hline Ed. Física & 2 & 8,7 & - & - & - & - & 2 & 3,9 \\
\hline Religión & - & - & - & - & 4 & 18,2 & 4 & 7,8 \\
\hline Ed. Párvulos & 1 & 4,3 & - & - & 2 & 9,1 & 3 & 5,9 \\
\hline Ed. Diferencial & - & - & - & - & 2 & 9,1 & 2 & 3,9 \\
\hline Artes Visuales & 1 & 4,3 & - & - & 1 & 4,5 & 2 & 3,9 \\
\hline Orientación & 1 & 4,3 & - & - & - & - & 1 & 2,0 \\
\hline Ed. Musical & - & - & - & - & 1 & 4,5 & 1 & 2,0 \\
\hline Inglés & - & - & - & - & 1 & 4,5 & 1 & 2,0 \\
\hline Total & 23 & 100 & 6 & 100 & 22 & 100 & 51 & 100 \\
\hline
\end{tabular}


En resumen, la muestra del estudio quedó compuesta por 51 profesionales de la educación, de distinto género y rangos de edad, egresados de distintas instituciones de educación superior y que se desempeñan como profesores de alguna de las 13 especialidades representadas en el estudio.

\section{ESTILOS DE GESTIÓN PEDAGÓGICA DE LOS PROFESORES DE LA MUESTRA}

Al examinar los resultados obtenidos en las cuatro escalas de pensamiento y actuación (Tabla 4), se encontró que los docentes obtienen mayores valores en la escala Conceptualización Abstracta (CA) con un valor promedio de 18,16 puntos y un máximo valor de 27 de los 28 puntos posibles. En la escala Experimentación Activa (EA) también se obtienen puntajes superiores con un promedio de 17,47 puntos. El menor valor promedio lo obtiene la escala Observación Reflexiva (OR) con 15,55 puntos, lo cual estaría expresando una menor atención hacia la observación rigurosa y reflexiva, en función de lograr una mejor comprensión antes de pasar a la acción. La escala Experiencia Concreta (EC) es la que presenta menor variabilidad en las puntuaciones obtenidas por los docentes.

Tabla 4. Resultados obtenidos por los profesores en las 4 escalas de orientación

\begin{tabular}{|c|c|c|c|c|}
\hline & $\begin{array}{c}\text { Experiencia } \\
\text { Concreta }\end{array}$ & $\begin{array}{c}\text { Observación } \\
\text { Reflexiva }\end{array}$ & $\begin{array}{c}\text { Conceptualización } \\
\text { Abstracta }\end{array}$ & $\begin{array}{c}\text { Experimentación } \\
\text { Activa }\end{array}$ \\
\hline $\mathrm{N}$ & 51 & 51 & 51 & 51 \\
\hline Media & 16,90 & 15,55 & 18,16 & 17,47 \\
\hline Mediana & 16,00 & 15,00 & 18,00 & 18,00 \\
\hline Moda & 16 & 13 & 20 & 18 \\
\hline $\begin{array}{c}\text { Desviación } \\
\text { típica }\end{array}$ & 3,028 & 3,360 & 3,408 & 3,355 \\
\hline Varianza & 9,170 & 11,293 & 11,615 & 11,254 \\
\hline Rango & 13 & 15 & 16 & 14 \\
\hline Mínimo & 11 & 9 & 11 & 10 \\
\hline Máximo & 24 & 24 & 27 & 24 \\
\hline Suma & 862 & 793 & 926 & 891 \\
\hline
\end{tabular}

Al sustraer los valores obtenidos en las escalas CA - EC y EA - OR, se identificó la distribución de los docentes al interior de los cuadrantes de estilos de gestión, encontrándose que mayoritariamente tienden a orientarse hacia el estilo pragmático $(41,2 \%)$ y minoritariamente $(7,8 \%)$ hacia el estilo creativo (Tabla 5). 11 profesores de la muestra $(21,6 \%)$ se situaron en el vértice o muy cercano al vértice de los cuatro cuadrantes, es 
decir, presentan un estilo de gestión pedagógico múltiple o integral pues no se alinean con ninguna de las cuatro orientaciones típicas.

Tabla 5. Distribución de los estilos de gestión de los docentes

\begin{tabular}{|c|c|c|}
\hline Estilos & Frecuencia & Porcentaje \\
\hline Analítico & 5 & 9,8 \\
\hline Pragmático & 21 & 41,2 \\
\hline Creativo & 4 & 7,8 \\
\hline Dinámico & 10 & 19,6 \\
\hline Múltiple & 11 & 21,6 \\
\hline Total & 51 & 100,0 \\
\hline
\end{tabular}

Al agrupar los estilos de acuerdo al hemisferio cerebral dominante en la actividad pedagógica, se encontró que el $51 \%$ se orienta preferentemente por los estilos de gestión vinculados al hemisferio izquierdo, tal como fue planteado en hipótesis del estudio (Tabla 6). Llama la atención la baja proporción de docentes orientados hacia formas analíticas de pensamiento y actuación $(9,8 \%)$ y la fuerza que tiene la orientación pragmática en los docentes de la muestra $(41,2)$. Esta situación que podría estar reflejando, por una parte, un distanciamiento de los docentes de la forma de pensamiento que fuera hegemónica a lo largo del siglo XX y, por otra, un alineamiento docente a las actuales demandas de la política educativa que exige obtener resultados de aprendizaje crecientes y medibles en los alumnos.

Por otra parte, una proporción importante de docentes $(27,4 \%)$ tiende a orientarse por formas de pensamiento y actuación que consideran componentes asociados al hemisferio izquierdo del cerebro. Asimismo, se encontró que el 21,6\% de los docentes de la muestra presenta un estilo de gestión polifacético o múltiple, es decir, tienen como característica desplazarse en su actividad educativa a través de procesos de pensamiento y actuación que consideran componentes de ambos hemisferios.

Tabla 6. Estilos de gestión y hemisferios dominantes en los docentes

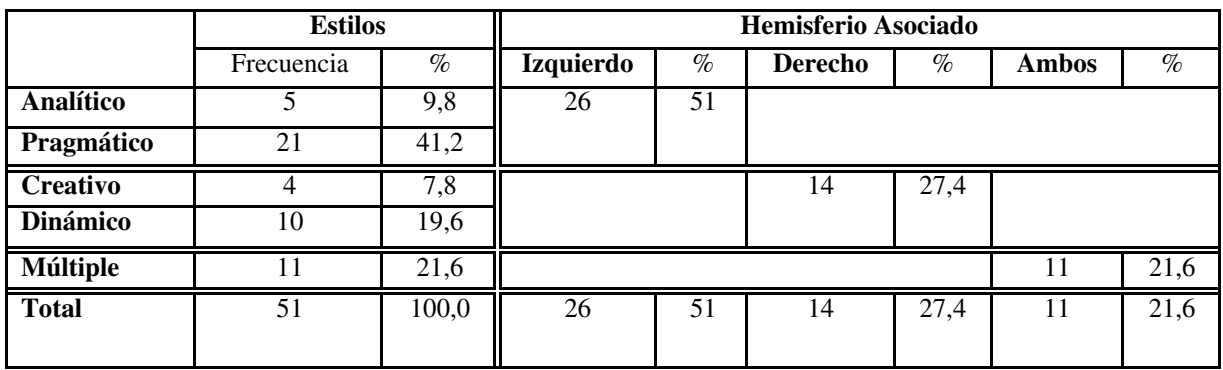


Comparando distribución de los estilos de gestión de acuerdo a la variable género, se observa que tanto profesoras como profesores presentan similar distribución en el estilo pragmático (Tabla 7). Asimismo, no se encuentran grandes diferencias por género en el estilo dinámico. Sin embargo, se observa que son los profesores los que tienden a orientarse hacia los estilos analítico o creativo y, por el contario, son profesoras las que tienden a orientarse por el estilo de gestión múltiple. El dato más relevante es que $90 \%$ de los docentes que tienen este estilo polifacético son profesoras, es decir, ellas tienen mayor disposición que los docentes varones a diseñar y aplicar estrategias pedagógicas holísticas que toman en cuenta, en algún grado, las cuatro formas típicas de pensamiento y actuación.

Tabla 7. Distribución de los estilos de gestión según género

\begin{tabular}{|c|c|c|c|c|c|c|}
\hline \multirow[t]{3}{*}{ Estilo } & \multicolumn{4}{|c|}{ Género } & & \\
\hline & \multicolumn{2}{|c|}{ Masculino } & \multicolumn{2}{|c|}{ Femenino } & \multicolumn{2}{|c|}{ TOTAL } \\
\hline & Frecuencia & $\%$ & Frecuencia & $\%$ & Frecuencia & $\%$ \\
\hline Analítico & 2 & 16,7 & 3 & 7,7 & 5 & 9,8 \\
\hline Pragmático & 5 & 41,6 & 16 & 41,1 & 21 & 41,2 \\
\hline Creativo & 2 & 16,7 & 2 & 5,1 & 4 & 7,8 \\
\hline Dinámico & 2 & 16,7 & 8 & 20,5 & 10 & 19,6 \\
\hline Múltiple & 1 & 8,3 & 10 & 25,6 & 11 & 21,6 \\
\hline Total & 12 & 100 & 39 & 100 & 51 & 100 \\
\hline
\end{tabular}

Con relación a la distribución de los estilos de gestión de acuerdo a rangos de edad (Tabla 8), se encontró que el estilo pragmático u orientado a resultados es la orientación predominante entre los docentes que están recién incorporados al sistema educativo $(53,8 \%)$, en desmedro de los estilos analítico, creativo y múltiple que tienen mayor presencia a medida que se avanza en los rangos de edad. El hecho que el segmento de docentes jóvenes tienda a ser pragmático, podría estar señalando que ellos tienen mayor apertura o menor resistencia a los cambios hacia la eficacia que propugna el sistema educacional actual.

Por otra parte, destaca que el estilo de gestión múltiple se concentre en los rangos de edades medianas, ya que el $72,7 \%$ de los 11 docentes que presentan ese estilo se ubica en los rangos de edades que fluctúan entre 31 y 50 años, lo cual podría estar insinuando la importancia que tendría la experiencia docente en avanzar hacia una gestión pedagógica más integral que considere funciones asociadas a ambos hemisferios. Asimismo, es probable que los docentes jóvenes tiendan a apoyarse en teorías o modelos rígidos en sus primeros años de ejercicio de la profesión, los cuales pueden ser flexibilizados o encontrar un modelo más equilibrado a medida que ganan experiencia. 
Tabla 8. Distribución de los estilos de gestión de acuerdo a rangos de edad

\begin{tabular}{|c|c|c|c|c|c|c|c|c|c|c|c|c|}
\hline \multirow{2}{*}{ Estilos } & \multicolumn{10}{|c|}{ Edad } \\
\cline { 2 - 15 } & $\begin{array}{c}\text { menos de } \\
\text { 30 años }\end{array}$ & $\begin{array}{c}\text { entre 31 y } \\
\mathbf{4 0} \text { años }\end{array}$ & $\begin{array}{c}\text { entre 41 y } \\
\mathbf{5 0} \text { años }\end{array}$ & $\begin{array}{c}\text { entre 51 y } \\
\text { 60 años }\end{array}$ & $\begin{array}{c}\text { más de 61 } \\
\text { años }\end{array}$ & \multicolumn{2}{c|}{ Total } \\
\hline & Frec. & $\%$ & Frec. & $\%$ & Frec. & $\%$ & Frec. & $\%$ & Frec. & $\%$ & Frec. & $\%$ \\
\hline Analítico & 1 & 7,7 & 2 & 18,2 & 2 & 13.3 & - & - & - & - & 5 & 9,8 \\
\hline Pragmático & 7 & 53,8 & 3 & 27.3 & 7 & 46.7 & 3 & 30 & 1 & 50 & 21 & 41,2 \\
\hline Creativo & 1 & 7,7 & 1 & 9,1 & - & - & 1 & 10 & 1 & 50 & 4 & 7,8 \\
\hline Dinámico & 3 & 23,1 & 2 & 18,2 & 1 & 6.7 & 4 & 40 & - & - & 10 & 19,6 \\
\hline Múltiple & 1 & 7,7 & 3 & 27.3 & 5 & 33.3 & 2 & 20 & - & - & 11 & 21,6 \\
\hline Total & 13 & 100 & 11 & 100 & 15 & 100 & 10 & 100 & 2 & 100 & 51 & 100 \\
\hline
\end{tabular}

Con relación a la distribución de los estilos de gestión de acuerdo el tipo de universidad de egreso (Tabla 9), se observa que el estilo de gestión dinámico se distribuye similarmente entre los distintos tipos de instituciones de egreso de los docentes de la muestra. Asimismo, el estilo de gestión pragmático se distribuye homogéneamente entre los egresados de universidades estatales $(43,5 \%)$ y privadas $(45,5 \%)$. El segmento de docentes que adhieren el estilo analítico proviene mayoritariamente $(75 \%)$ de universidades estatales, al igual que los docentes que presentan el estilo creativo (60\%). Los docentes que tienden a orientarse por el estilo de gestión múltiple son egresados de universidades privadas del CRUCh $(50 \%)$ o privadas $(27,3 \%)$.

Reconociendo que hay una gran cantidad de factores personales, globales e institucionales que influyen en el tipo de orientación que presenta cada profesor, estos datos podrían estar señalando la importancia fundamental que tiene la formación inicial docente para el posterior desempeño, lo cual se va a reflejar en diversos aspectos como el conocimiento pedagógico adquirido, el dominio de las materias a tratar, la capacidad para comprender y aplicar didácticas específicas acordes con el sujeto de la educación y los programas ministeriales y, en este caso, el tipo de orientación hacia la actividad docente.

Tabla 9. Distribución de los estilos de gestión de acuerdo a universidad de egreso

\begin{tabular}{|c|c|c|c|c|c|c|c|c|}
\hline \multirow{2}{*}{ Estilo } & \multicolumn{6}{|c|}{ Tipo de universidad } & \multicolumn{2}{c|}{} \\
\cline { 2 - 10 } & \multicolumn{2}{|c|}{ Estatal } & \multicolumn{2}{|c|}{$\begin{array}{c}\text { Privada } \\
\text { Cruch }\end{array}$} & \multicolumn{2}{c|}{ Privada } & \multicolumn{2}{c|}{ Total } \\
& & & & & & & \\
\hline & Frec. & $\%$ & Frec. & $\%$ & Frec. & $\%$ & Frec. & $\%$ \\
\hline Analítico & 3 & 13 & 1 & 16,7 & 1 & 4,5 & 5 & 9,8 \\
\hline Pragmático & 10 & 43,5 & 1 & 16,7 & 10 & 45.5 & 21 & 41,2 \\
\hline Creativo & 3 & 13 & 0 & & 1 & 4,5 & 4 & 7,8 \\
\hline Dinámico & 5 & 21,7 & 1 & 16,7 & 4 & 18,2 & 10 & 19,6 \\
\hline Múltiple & 2 & 8,7 & 3 & 50 & 6 & 27,3 & 11 & 21,6 \\
\hline Total & 23 & 100 & 6 & 100 & 22 & 100 & 51 & 100 \\
\hline
\end{tabular}




\section{CONSIDERACIONES FINALES}

Este estudio exploratorio, realizado con 51 profesionales vinculados a siete instituciones educativas de la Región Metropolitana, encontró que los profesores se orientan por diversas formas de pensamiento y se distribuyen entre distintas modalidades de gestión. Sin embargo, se constató que la mayoría de los docentes se localiza en los cuadrantes asociados al hemisferio izquierdo del cerebro, donde predominan las funciones relacionadas con el razonamiento lógico y la abstracción, tal como fue planteado en la hipótesis. En esta situación destaca la fuerte presencia que tiene la orientación hacia resultados o pragmática en desmedro de la orientación analítica, lo cual puede ser interpretado como una respuesta de los docentes hacia las actuales demandas de la política educativa que propugnan la eficacia escolar. Este tipo de orientación, que pone énfasis en la organización, planificación y búsqueda de resultados, está igualmente presente en profesoras y profesores y es la orientación predominante en los docentes menores de 30 años.

En cuanto a las dos formas de gestión asociadas al hemisferio derecho, es el estilo socio-dinámico el que obtuvo mayor presencia y es representada mayoritariamente por profesoras, lo cual señala que existen diferencias genéricas para implicarse emocionalmente. El estilo de gestión creativo es el que tiene menor presencia, no presentando diferencias de género ni edad, pero sí en relación a universidad de egreso, ya que tres de los cuatro docentes creativos presentes en la muestra provienen de universidades estatales. La situación deficitaria de este estilo estaría señalando, por una parte, una gran debilidad de los profesores para responder adecuadamente a los permanentes desafíos de transformación institucional e innovación pedagógica y, por otra, la necesidad de actualizar la formación docente para atender al desarrollo de habilidades asociadas al pensamiento divergente, destrezas que son críticas para responder adecuadamente a las demandas de la sociedad del conocimiento hacia la educación.

El hecho que el 27,4\% de los profesores de la muestra adhieran al estilo de gestión dinámico o creativo puede estar indicando un proceso de gradual abandono la visión de la escuela como espacio de mera instrucción técnico-cognitiva y, por ende, la existencia de una mayor inclinación a visualizarla como un lugar de convivencia humana donde se deben considerar otras dimensiones de la experiencia del ser, las cuales han recibido poca consideración en los procesos educativos tradicionales.

Finalmente, el análisis de los datos permitió identificar un grupo constituido por el $21,6 \%$ de los profesores de la muestra que se orienta por un estilo de gestión múltiple, es decir, son docentes que no enfatizan una sola orientación y, en consecuencia, tienen la capacidad de desplazarse gradualmente por las cuatro orientaciones típicas. En esta orientación, la variable género resultó ser significativa, ya que el $91 \%$ de los docentes que presentan este estilo son profesoras. Asimismo, se encontró que el $92 \%$ de los docentes con estilo polifacético o múltiple son mayores de 30 años de edad, lo cual podría estar insinuando la importancia que tiene la experiencia docente en avanzar hacia la elaboración de estrategias pedagógicas más versátiles e integrales, es decir, utilizando formas de pensamiento y actuación de todo tipo. Además, se encontró que el sólo 8,7\% de los docentes que tienen este estilo proviene de universidades estatales, lo cual podría estar señalando las limitaciones que han tenido estas instituciones, por razones estructurales, para incorporar innovaciones curriculares y pedagógicas en la formación docente que permitan el desarrollo de habilidades académicas y personales por sobre los contenidos. 
Desde la perspectiva de la gestión pedagógica, cuyo objetivo es centrar y dinamizar la práctica docente alrededor del desarrollo integral de los alumnos, la orientación múltiple es la que mejor se ajusta al nuevo escenario de las sociedades postindustriales donde los docentes deberán asumir el rol de dinamizadores de procesos de producción y gestión de conocimiento, y para esto deberán poseer y articular las diversas orientaciones y habilidades de gestión pedagógica. Además, los docentes que posean este perfil versátil podrán hacer un valioso aporte a los procesos de formación de personas para el siglo $\mathrm{XXI}$, puesto que estas profesoras y profesores polifacéticos utilizarán diversas estrategias, metodologías y didácticas, dirigidas al desarrollo integral de sus alumnos, en toda su realidad humana, es decir, formar personas con habilidades intelectuales, creativas, valóricas, emocionales y prácticas.

\section{REFERENCIAS BIBLIOGRÁFICAS}

Agramonte, R. (1991). Las doctrinas educativas y políticas de Martí. Puerto Rico: Editorial de la Universidad de Puerto Rico.

Alighiero, M. (1976). Historia de la Educación. Tomo 2. México: Ed. Siglo XXI.

Aparici, R. (1996). La Revolución de los medios audiovisuales: educación y nuevas tecnologías. España: Ediciones de la Torre.

Bastías, M. (2011). Hacia nuevas prácticas educativas en la sociedad del conocimiento. Revista Temas Pedagógicos, n. 13, 271-283.

Brunner, J. J. (2000). Nuevos escenarios de la educación. Revolución tecnológica y Sociedad de la Información. Santiago: PREAL.

Carretero, M. (2005). Constructivismo y educación. México: Editorial Progreso.

Comte, A. (1982). Discurso sobre el espíritu positivo. Argentina: Editorial Aguilar.

De Bono, E. (1994). El pensamiento creativo: el poder del pensamiento lateral para la creación de nuevas ideas. Barcelona: Paidós.

Dilthey, W. (1978). Introducción a las ciencias del espíritu. México: Ediciones Fondo de Cultura Económica.

Echeverria, R. (2008). Actos de lenguaje. Buenos Aires: Ediciones Granica.

Ferraris, M. (2002). Historia de la hermenéutica. México: Siglo XXI.

Guilford, J. P.(1983). Creatividad y educación. Barcelona: Paidós Ibérica.

Goleman, D. (1997). La inteligencia emocional. Buenos Aires: Javier Vergara.

Hanushek, E. (2007). Calidad de la Educación y Crecimiento Económico. Santiago: Documento $\mathrm{N}^{\circ} 39$, PREAL.

Kolb, D. (1984). Experiential learning: experience as the source of learning and development. New York: Prentice Hall, Englewood Cliffs.

Morgan, G. (1996). Imágenes de la organización. México: Alfaomega Grupo Editor.

Morin, E. (1996). El pensamiento complejo contra el pensamiento único. En Sociología y Política, a. IV, n. 8.

Nonaka, I. y Takeuchi, H. (1999). La organización creadora del conocimiento: Cómo las compañías japonesas crean la dinámica de la innovación. New York: Oxford University Press.

OECD (2009). La comprensión del cerebro: El nacimiento de una ciencia del aprendizaje. Santiago: Universidad Católica Silva Henríquez.

Palacios, J. (1991). La educación en el siglo XX (I): La tradición renovadora. Caracas: Cooperativa Laboratorio Educativo.

Popkewitz, T. (1990). Formación del profesorado: Tradición, teoría y práctica. España: Universidad de Valencia. 
Rodríguez, M. (2003). La metamorfosis del cambio educativo. España: Ediciones AKAL. Salas, R. (2008). Estilos de aprendizaje a la luz de la neurociencia. Bogotá: Magisterio. SEP (2004). Manual de estilos de aprendizaje. México: DGB/DCA.

Taylor, F. (1981). Principios de la administración científica. Buenos Aires: El Ateneo. Toffler, A. (1980). La tercera ola. Barcelona: Esplugues de Llobregat, Plaza \& Janés. UNESCO (1994). Hacia las sociedades del conocimiento. París: UNESCO.

Verlee, L. (1995). Aprender con todo el cerebro. España: Ed. Martínez Roca.

Weber, M. (1996). Economía y sociedad. México: Ediciones Fondo de Cultura Económica. 